\title{
Eyes wide open
}

\author{
Understanding how proteins function in isolation and in their native context requires a merging of molecular-level \\ techniques that explore the interplay of protein structure and dynamics.
}

$T_{\text {himing }}^{\mathrm{h}}$ he concepts of 'one gene, one enzyme' and 'structure determines function' are basic tenets of modern biochemistry. Add to this the idea that organisms are machines that can be understood as a sum of their parts, and you have the makings of a reductionist viewpoint encompassing the implied hierarchy of physics ruling chemistry and chemistry ruling biology. Yet it is clear that enzymes and proteins are inherently motion-filled structures that, more often than not, act in complexes rather than alone. Understanding not only how proteins behave in isolation but also how they recognize their binding partners and act in this context is therefore critical for a full understanding of protein function. In this issue, we highlight insights gained in the study of protein dynamics - the forces, mechanics and motions that identify proteins as the workhorses of biology.

From the theoretical framework of reductionism, much has been learned about biological pathways and how proteins in them are regulated. High-resolution methods have also provided great insights into the molecular-level details of key biological processes, including the mechanisms by which proteins fold and interact with one another, the signals necessary for marking a protein for degradation (Review, p. 815 ), and the kinetic principles of biochemical reactions. The fact that we can design protein-protein interaction interfaces points to a wellhoned understanding of the principles of protein folding and molecular recognition (Review, p. 797). Although much work remains to be done to gain a full accounting of the basic principles of molecular function, these concepts have provided a critical platform from which to launch investigations into the more complex global issues of biology that extend beyond its component parts.

In their Commentary (p. 774), Gierasch and Gershenson embrace such an integrated perspective by arguing that the 'holy grail' of dissecting the function of proteins is to study them in situ. This "post-reductionist" view marks a different way of thinking about the approach to biological problems and reminds us of the strengths of scientific inquiry: the precise framing of problems, the clarity to understand what is and what is not the truth and the importance of hypothesis-driven research. Fully comprehending the workings of a cell will require understanding the dynamics and interplay between all of the parts and pathways. Knowing the parts of isolated entities and viewing them through "eyes-down" spectacles is not enough. Cells are not machines-they are more than the sum of their parts.

A focus on more complex problems such as proteostasis at a cellular level or stem cell regeneration at an organismal level does not require eschewing the traditional molecular methodology. Rather, it highlights the importance of placing the system in its proper biological context. There is still much work to do to know enough about the molecular (that is, chemical and physical) interactions at the most fundamental levels and to develop precise methods that can fully explain complex phenomena. So, a reductionist toolbox is still needed. But reductionism has sometimes overlooked other biological fields, for instance in the more "eyes-up" study of human physiology or even in understanding the resistance to antibiotics among different bacteria, and a more complete understanding requires taking a page from sociology, where ideas from the micro level are used to understand and model what happens in a larger societal context.

A broader, more modern goal of unifying physics and biology is captured in the idea that an ensemble of conformations of a given protein exists at any given time, awaiting selection by a binding partner (Perspective, p. 789). In contrast to the classical 'induced-fit' model for molecular recognition, the 'conformational selection' model takes into account the dynamics of proteins at a whole-cell level. This idea, combined with recent work demonstrating the existence and function of proteins without a well-defined three-dimensional structure (known as intrinsically disordered proteins), challenges our most central ideas about the dependence of structure and function. The fact that these proteins exist, and that they are involved in myriad processes including transcriptional regulation and signaling, supports the ideas of Baldwin and Kay that the "interplay between structure and dynamics determines both the function of a protein and its ability to evolve and adapt to a diverse set of conditions" (see p. 778 and p. 808). Protein dynamics are integrally connected with protein structure in determining function.

From the notion that a protein's function is determined by its structure and dynamics, it is clear that physical models and methods are essential for a full understanding of protein function. Because of the role that physical chemistry plays in the details of protein motionsfor example, active sites opening or amino acid side chains shifting their positions within a protein structure-both experimental and theoretical studies within this field are poised to contribute to a full understanding of protein function. In our view, studies that are rich in mechanistic insight (including those more traditionally defined by the principles and methods of biophysics) are a key component of current chemical biology research. However, we also feel that such studies are significantly strengthened by complementary insights into the biological system under study. Just as proteins should be considered as dynamic, non-isolated entities, the merging of scientific disciplines either within one research laboratory or through collaboration is integral in understanding biology at the comprehensive level.

Many examples exist where insights learned from high-resolution studies of component parts in vitro have led to discoveries in vivo, so working at this level remains an important and fruitful arena of research. Emergent fields such as systems biology and synthetic biology are based upon integrating the finer details of biology into lifesized models. Working within the context of a whole cell, a tissue or even an organism poses a great set of challenges both intellectual and technical, but such an "eyes-up" approach is necessary to gain a full "eyes-open" understanding of biology. 